\title{
A NOTE ON STRATIFIABLE SPACES ${ }^{1}$
}

\section{R. A. STOLTENBERG}

1. Introduction. The class of stratifiable spaces has been shown to possess many important properties (see for example [2] and [3]), and the purpose of this note is to give two additional properties that stratifiable spaces have. The definitions of the terms used in this paper are given below.

The following theorem generalizes a result of Ceder's [3] and answers a question raised by Arhangel'skiì on p. 142 of [1].

1.1. Theorem. Let $\nu$ be any cardinal number. For a stratifiable space $X$ the following are equivalent:

(a) There is a subset of cardinality less than or equal to $\nu$ which is dense in $X$,

(b) Each open cover $\mathfrak{U}$ has a subcover of cardinality less than or equal to $\nu$,

(c) Every disjoint system of open sets has cardinality less than or equal to $\nu$,

(d) X has a network ${ }^{2}$ of cardinality less than or equal to $\nu$.

Several versions of the following theorem have appeared in recent years, many of them are mutually incomparable [1, Theorems 4.3 4.7], [5], [6], [7, Theorem 1]. Our theorem simultaneously generalizes results of Lašnev [6] (where $X$ is metrizable), Arhangel'skii [1, Theorem 4.7] (where $X$ has a countable network), and Okuyama [7] (where $X$ a normal $\sigma$-space).

1.2. Theorem. If $X$ is a normal semistratifiable space and $f: X \rightarrow Y$ is a continuous closed surjection, then $Y=Y^{\prime} \cup Y^{\prime \prime}$ where $Y^{\prime}$ is a $\sigma$-discrete subset of $Y$ and $f^{-1}(y)$ is compact for each $y \in Y^{\prime \prime}$.

Let $X$ be a topological space (we will assume all topological spaces to be $T_{2}$ ). A semistratification for $X$ assigns to each open $U \subset X$ a sequence of closed sets $\left\{U_{n} \mid n=1,2, \cdots\right\}$ such that:

(i) $U_{n} \subset V_{n}$ whenever $U$ and $V$ are open in $X$ and $U \subset V$.

(ii) $U_{n-1}^{\infty} U_{n}=U$.

Presented to the Society, January 24, 1969; received by the editors October 13, 1968.

1 The author wishes to thank the referee for his helpful comments and criticisms.

${ }^{2}$ A collection $\mathscr{N}$ is challed a network for $X$ if and only if for each $x \in X$ and each open subset $U$ of $X$ containing $x$ there is $N \in \mathscr{N}$ such that $x \in N \subset U$ (Compare [1]). 
A stratification for $X$ is a semistratification with the additional property:

(iii) $\cup_{n-1}^{\infty} U_{n}^{0}=U$ where $U_{n}^{0}$ denotes the interior of $U_{n}$.

A $T_{1}$ space $X$ is called a (semi) stratifiable space if there exists a (semi) stratification for $X$. Clearly we may assume that a (semi) stratification for $X$ is increasing, i.e. $U_{n} \subset U_{n+1}$ for each $n$.

It is clear from the definition that a stratifiable space is semistratifiable and it is easily checked that $\sigma$-spaces in the sense of Okuyama [7] (i.e. spaces with a $\sigma$-locally finite network) are also semistratifiable. Hence the conclusion of Theorem 1.2 is valid for all stratifiable spaces (which are automatically normal by [3]) and for all normal $\sigma$-spaces. The latter observation strengthens a result of Okuyama [7].

In [3] Ceder notes that (a), (b) and (c) of Theorem 1.1 are equivalent whenever $\nu \leqq \boldsymbol{\aleph}_{0}$ and under the same assumption Michael proved that (b) and (d) are equivalent. Later in [1] Arhangel'skiì observed that (b) and (d) are equivalent for any cardinal number. When originally submitted, this paper contained a direct (but rather lengthy) proof of the equivalence of (a), (b) and (c) for any cardinal number. Since then, however, R. W. Heath has succeeded in proving that every stratifiable space is a $\sigma$-space. Since the equivalence of (a), (b), (c), and (d) is essentially trivial for paracompact $\sigma$-spaces, Theorem 1.1 now follows immediately from Heath's theorem and the fact that stratifiable spaces are paracompact [3].

2. Proof of Theorem 1.2. In a topological space $X$ a sequence of sets $\left\{B_{n} \mid n=1,2, \cdots\right\}$ converges to a set $B$ contained in $X$ if for every neighborhood $V$ of $B$ there is a positive integer $n$ such that $B_{n} \subset V$ for all $n \geqq n_{0}$.

The following lemma slightly sharpens a result of Lašnev [6].

2.1. Lemma. Let $X$ be a normal space. If a sequence $\left\{B_{n} \mid n=1,2, \cdots\right\}$ of closed subsets of $X$ converges to a closed set $B$ such that $B \cap B_{n}=\varnothing$ for each $n$, then $C=B \cap\left(\cup_{n}^{\infty}{ }_{-1} B_{n}\right)-i$ countably compact.

Proof. We will show that every sequence $\left\{x_{k} \mid k=1,2, \cdots\right\}$ in $C$ has an accumulation point. Suppose this is not so. Let $\left\{x_{k} \mid k=1,2, \ldots\right\}$ be a sequence in $C$ which has no accumulation points. Then $\left\{x_{k} \mid k=1,2, \cdots\right\}$ is a closed discrete set in $X$. Since $X$ is normal there is an open set $U_{k}$ such that $x_{k} \in U_{k}$ for each $k$ and $\left\{U_{k} \mid k=1,2, \cdots\right\}$ is discrete. Since $x_{k} \in\left(\cup_{n=1}^{\infty} B_{n}\right)^{-}$it follows that $U_{k} \cap B_{n} \neq \varnothing$ for infinitely many $n$. So there is a sequence $\left\{n_{k} \mid k=1,2, \cdots\right\}$ such that $n_{k}<n_{k+1}$ and $U_{k} \cap B_{n_{k}} \neq \varnothing$. Let 
$y_{k} \in U_{k} \cap B_{n_{k}}$, then the set $S=\left\{y_{k} \mid k=1,2, \cdots\right\}$ is discrete and hence closed in $X$. So $X-S$ is an open neighborhood of $B$. However, there is no positive integer $n_{0}$ such that $B_{n} \subset X-S$ for $n \geqq n_{0}$ contrary to our hypothesis. Hence our assumption that $\left\{x_{k} \mid k=1,2, \cdots\right\}$ does not have an accumulation point is false. So $C$ is countably compact.

Proof of Theorem 1.2. Let $A(y)=f^{-1}(y)$ for each $y \in Y$ and let $V(y)=X-A(y)$. Since $V(y)$ is open in $X$ there is a semistratification $\left\{V_{n}(y) \mid n=1,2, \cdots\right\}$ for $V(y)$. Let $U(y, n)=X-V_{n}(y)$ for each $n$ and each $y \in Y$. Clearly $A(y) \subset U(y, n)$ and hence there is an open neighborhood $O(y, n)$ of $y$ such that $f^{-1}(O(y, n)) \subset U(y, n)$ for each $n$. Let $Y_{n}=\left\{y \mid y \notin O\left(y^{\prime}, n\right)\right.$ for any $y^{\prime} \neq y$ in $\left.Y\right\}$. Then $Y_{n}$ is a discrete subset of $Y$ for each $n$ : Let $B \subset Y_{n}$ and let $y \in\left(\cup\left\{y^{\prime} \mid y^{\prime} \in B\right\}\right)^{-}$. Thus $O(y, n) \cap\left(\cup\left\{y^{\prime} \mid y^{\prime} \in B\right\}\right) \neq \varnothing$ and hence $y^{\prime} \in O(y, n)$ for some $y^{\prime} \in B$ $\subset Y_{n}$. Thus it follows from the definition of $Y_{n}$ that $y=y^{\prime}$. So $Y_{n}$ is closure preserving and thus discrete.

Let $Y^{\prime}=\cup_{n-1}^{\infty} Y_{n}$ and let $Y^{\prime \prime}=Y-Y^{\prime}$. Suppose $y \in Y^{\prime \prime}$; then for any $n$ there is $y_{n} \in Y$ such that $y \in O\left(y_{n}, n\right)$. We first show that $A(y) \subset\left(\bigcup_{n_{-1}^{\infty}}^{\infty} A\left(y_{n_{k}}\right)\right)$ - for any monotone increasing sequence of positive integers $\left\{n_{k} \mid k=1,2, \cdots\right\}$. Suppose not; then there is an $x \in A(y)-\left(\bigcup_{k-1}^{\infty} A\left(y_{n_{k}}\right)\right)^{-}$. Let $U=X-\left(\bigcup_{k_{-1}^{\infty}} A\left(y_{n_{k}}\right)\right)^{-}$and let $k$ be the first positive integer such that $x \in U_{k}$. Clearly $U \subset X-A\left(y_{n_{k}}\right)$ $=V\left(y_{n_{k}}\right)$ and hence $U_{n_{k}} \subset V_{n_{k}}\left(y_{n_{k}}\right)$. Thus

$$
f^{-1}\left(O\left(y_{n_{k}}, n_{k}\right)\right) \subset U\left(y_{n_{k}}, n_{k}\right)=X-V_{n_{k}}\left(y_{n_{k}}\right) \subset X-U_{n_{k}} .
$$

However, $y \in O\left(y_{n_{k}}, n_{k}\right)$ and hence $A(y) \subset U\left(y_{n_{k}}, n_{k}\right)$. So

$$
\{x\} \subset U_{k} \cap A(y) \subset U_{n_{k}} \cap A(y)=\varnothing,
$$

a contradiction.

Now let $V$ be any open neighborhood of $A(y)$. Then there is an open neighborhood $O(y)$ of $y$ such that $f^{-1}(O(y))=U(A(y)) \subset V$. If there is a monotone increasing sequence $\left\{n_{k} \mid k=1,2, \cdots\right\}$ of positive integers such that $A\left(y_{n_{k}}\right) \nsubseteq U(A(y))$ then $U(A(y)) \cap A\left(y_{n_{k}}\right)=\varnothing$ for each $k$. Thus $U(A(y)) \cap\left(\bigcup_{k_{-1}^{\infty}}^{\infty} A\left(y_{n_{k}}\right)\right)=\varnothing$ and hence $A(y)$ $\Phi\left(\bigcup_{k-1}^{\infty} A\left(y_{n_{k}}\right)\right)$-, contrary to what was proven above. Thus $\left\{A\left(y_{n}\right) \mid n=1,2, \cdots\right\}$ converges to $A(y)$. So it follows from Lemma 2.1 that $A(y) \cap\left(\cup_{n=1}^{\infty} A\left(y_{n}\right)\right)^{-}=A(y)$ is countably compact in $X$ and hence it follows from Corollary 4.5 in [4] that $A(y)$ is compact in $X$. This completes the proof of Theorem 1.2.

We conclude this note with the following observations on Theorem 1.2:

2.2. REMARK. (a) For each $y \in Y^{\prime \prime}$ we have that $f^{-1}(y)=A(y)$ has an empty interior. This follows from the proof of Theorem 1.2 for 
we proved that $A(y) \cap\left(\bigcup_{n=1}^{\infty} A\left(y_{n}\right)\right)^{-}=A(y)$ for some sequence $\left\{y_{n} \mid n=1,2, \cdots\right\} \subset Y$ where $y_{n} \neq y$ for any $m$. Hence $A(y)$ $\subset\left(\cup_{n=1}^{\infty} A\left(y_{n}\right)\right)^{-}-\bigcup_{n=1}^{\infty} A\left(y_{n}\right)$ and so $A(y)^{0}=\varnothing$.

(b) The cardinality of $Y^{\prime}$ is less than equal to the weight $\nu$ of $X$. This follows from the fact that $Y$ has a network of weight $\nu$. So there can not be a discrete set in $Y$ of cardinality larger than $\nu$.

\section{REFERENCES}

1. A. V. Arhangel'skiř, Mappings and spaces, Uspehi. Mat. Nauk 21 (1966), 133184 = Russian Math. Surveys 21 (1966), 115-162.

2. C. J. R. Borges, On stratifiable spaces, Pacific J. Math. 17 (1966), 1-16.

3. J. G. Ceder, Some generalization of metric spaces, Pacific J. Math. 11 (1961), 105-126.

4. G. Creede, Semi-stratifiable spaces, Topology Conference, Arizona State University, Temple, Arizona, 1967, pp. 318-324.

5. V. V. Filippov, On feathered paracompacta, Dokl. Akad. Nauk SSSR 178 (1968), 555-558 = Soviet Math Dokl. 9 (1968), 161-164.

6. N. S. Lašnev, Continuous decompositions and closed mapping of metric spaces, Dokl. Akad. Nauk. SSSR 165 (1965), 756-758 = Soviet Math. Dokl. 6 (1965), 15041506.

7. A. Okuyama, $\sigma$-spaces and closed mappings. I, Proc. Japan Acad. 44 (1968), $472-477$.

Washington State University 